\title{
On the neutral gas distribution and kinematics in the dwarf irregular galaxy IC 1613
}

\author{
S. Silich ${ }^{1,7}$, T. Lozinskaya ${ }^{2}$, A. Moiseev ${ }^{3}$, N. Podorvanuk ${ }^{2}$, M. Rosado ${ }^{4}$, J. Borissova ${ }^{5}$, and M. Valdez-Gutierrez ${ }^{6}$ \\ ${ }^{1}$ Instituto Nacional de Astrofísica Optica y Electrónica, AP 51, 72000 Puebla, México \\ e-mail: silich@inaoep.mx \\ 2 Sternberg Astronomical Institute, Universitetskii pr. 13, Moscow, 119992, Russia \\ e-mail: lozinsk@sai.msu.ru \\ 3 Special Astrophysical Observatory, Russian Academy of Sciences, Nizhnii Arkhyz, 357147 Karachai-Cherkessian Republic, Russia \\ e-mail: moisav@sao.ru \\ 4 Instituto de Astronomía, Universidad Nacional Autónoma de México, AP 70-264, 04510 México DF, México \\ e-mail: margarit@astroscu.unam.mx \\ 5 European Southern Observatory, Ave., Alonso de Cordova 3107, Casilla 19 Santiago 19001, Chile \\ e-mail: jborisso@eso.org \\ ${ }^{6}$ Instituto de Astronomía, UNAM, AP 877, Ensenada 22800, B.C., México \\ e-mail: mago@astrosen.unam.mx \\ 7 Visiting professor in the Department of Physics and Astronomy at the University of Kentucky, USA
}

Received 28 April 2005 / Accepted 13 October 2005

\section{ABSTRACT}

Aims. We study the neutral hydrogen distribution and kinematics in the Local Group dwarf irregular galaxy IC 1613 and compare them with the ionized gas distribution and stellar content of the galaxy. We discuss several mechanisms which may be responsible for the origin of the observed complicated HI morphology and compare parameters of the most prominent kpc-scale HI structure with the multiple SNe scenario. Methods. The observations were performed with the Vary Large Array of NRAO with a linear resolution $\sim 23$ pc at the adopted distance of $725 \mathrm{kpc}$ and the spectral channel width of $2.57 \mathrm{~km} \mathrm{~s}^{-1}$. The numerical calculations have been provided with our 2.5D Lagrangian scheme based on the thin layer approximation.

Results. We found that the ISM of the galaxy is highly inhomogeneous and identified a number of intermediate-scale (200 pc-300 pc in diameter) $\mathrm{HI}$ arcs and shells having expansion velocities of 10 to $20 \mathrm{~km} \mathrm{~s}^{-1}$. Besides these shells, several giant holes and arc-shaped structures have been revealed, whose radii exceed several hundred parsecs. We found that parameters of the most prominent $\left(M_{\mathrm{HI}}=2.8 \times 10^{7} M_{\odot}\right)$ kpc-scale structure and the level of the detected star formation activity are inconsistent with the multiple SNe hypothesis.

Key words. galaxies: irregular - Galaxy: kinematics and dynamics - ISM: structure - ISM: bubbles

\section{Introduction}

The neutral component of the ISM in star-forming dwarf and spiral galaxies presents a collection of holes, arcs, and shell-like structures (Davies \& Tovmassian 1963; Heiles 1984; Brinks \& Bajaja 1986; Puche et al. 1992; Ehlerová et al. 2004). Recent studies of late-type dwarf galaxies indicate that $\sim 1 / 3$ of the galaxies present a high column density and sometimes broken rings with $N_{\mathrm{HI}}>10^{21} \mathrm{~cm}^{-2}$ (Stil \& Israel 2002). The origin of HI holes and shell-like structures has been the subject of debate for more than two decades, but still remains a controversial issue (see, for example, Block \& Walter 2005).

Several different mechanisms have been proposed for the formation of the observed $\mathrm{HI}$ distribution and kinematics in different galaxies (see, for example, reviews by Tenorio-Tagle \& Bodenheimer 1988; van der Hulst 1996).

In the standard approach based on the Dyson \& de Vries (1972), Weaver et al. (1977) model, HI shells result from the cumulative effects of multiple stellar winds and supernovae explosions inside the volume presently encompassed by the shell. In such a case the energy released by stellar winds and supernovae is thermalized and generates a hot, high pressure cavity that drives a shock wave into the surrounding interstellar medium (ISM). This shock collects interstellar gas and compresses it into a dense, expanding shell. As the hot cavity continues to expand, the temperature and pressure inside the cavity drop while the outer shell decelerates. When the pressure becomes comparable to that of the ambient medium, 
the shock wave vanishes. At such a pressure-confined stage (Koo \& McKee 1992), the shell stalls and starts to disintegrate with its local sound speed.

Despite many cases in which this approach gives a plausible explanation for the observed structures (see, for example, Oey \& García-Segura 2004; Vorobyov et al. 2004, 2005; van der Hulst 1996, and references therein), it was recognized long ago (Heiles 1984; Tenorio-Tagle \& Bodenheimer 1988) that this scenario cannot explain all types of observed structures. In particular, it encounters problems when attempting to explain the origin of the largest supershells where the stellar associations found within the HI rings seem to be inadequate energy sources. More recently, Rhode et al. (1999) found that in the case of the Holmberg II (HoII) galaxy, the observed upper limits for stellar cluster remnants detected inside neutral hydrogen holes is in many cases inconsistent with the SNe hypothesis. Although a year later Stewart et al. (2000) re-examined their results using deep far-ultraviolet (FUV), $\mathrm{H} \alpha$ and $\mathrm{HI}$ data and decided that the energy deposited by stellar winds and $\mathrm{SNe}$ explosions into the ISM of HoII is sufficient, there are many HI holes that have no optical counterparts consistent with the rate of mechanical energy required by the standard model.

In other galaxies, Kim et al. (1999) found only a weak correlation between the LMC neutral hydrogen holes and the HII regions and concluded that the hypothesis of multiple winds and supernovae is inconsistent with their data. Perna $\&$ Gaensler (2004) proposed a non-traditional approach to the problem by counting the number of radio pulsars associated with Milky Way supershells. They performed Monte-Carlo simulations for the expected pulsar population and predicted that several tens of radio pulsars may be detected in the directions to the largest Milky Way supershells, and few of them should be found inside regions encompassed by the contours of smaller shells. They found that for the smaller shells their predictions are consistent with the detected pulsar population. However for the giant supershell GSH242-03+37 the observed number of radio pulsars is inconsistent with the multiple winds and supernovae model.

Several other mechanisms that do not require violent stellar activity include collisions of high velocity clouds with galactic disks (Tenorio-Tagle 1981), the radiation pressure from field stars (Elmegreen \& Chiang 1982), the non-linear evolution of self-gravitating turbulent galactic disks (Wada et al. 2000; Dib $\&$ Burkert 2004), the ram pressure of the intergalactic medium (Bureau \& Carignan 2002), and even exotic mechanisms such as the distortion of the ISM by powerful gamma-ray bursts (Efremov et al. 1999). Despite many efforts attempting to resolve this problem, the detailed comparison of different scenarios with objects detected in different galaxies remain scarce and controversial.

IC 1613 is a member of the Local Group and is located 725-730 kpc from the Milky Way (Freedman 1988a,b; Dolphin et al. 2001). Because of its proximity, it represents an excellent target to study the interplay among the stellar and gaseous components in galaxies. Its HI mass, derived from single-dish observations by Lake \& Skillman (1989), is $6 \times 10^{7} M_{\odot}$. The galactic rotation is slow, with an almost linear rotation curve inside the inner $2.5 \mathrm{kpc}$ radius, having a maximum amplitude of rotation velocity around $25 \mathrm{~km} \mathrm{~s}^{-1}$ (Lake \& Skillman 1989).

The stellar population of IC 1613 and its star formation history $(\mathrm{SFH})$ have been studied by a number of authors. Hunter et al. (1993) estimated the total $\mathrm{H} \alpha$ luminosity to be $L_{\mathrm{H} \alpha} \approx$ $3 \times 10^{38} \mathrm{erg} \mathrm{s}^{-1}$. The associated star formation rate (SFR) is in good agreement with that obtained by Cole et al. (1999), who found $S F R \approx 3 \times 10^{-3} M_{\odot} \mathrm{yr}^{-1}$ over the past $10 \mathrm{Myr}$. Skillman et al. (2003) did not find any evidence for recent episodes of violent star formation in this galaxy from their analysis of colormagnitude diagrams and concluded that it looks unlikely that this and similar galaxies may be responsible for the chemical enrichment of the intergalactic medium. Georgiev et al. (1999) obtained deep $U B V$ photometry for more than 3000 stars in selected areas of IC 1613. The isochrone fit of their data clearly indicates the presence of young (age $<30 \mathrm{Myr}$ ) stars and stellar associations.

On the other hand, Fabry-Perot interferometric studies of the ionized gas kinematics (Valdez-Gutiérrez et al. 2001; Lozinskaya et al. 2003) revealed a number of supershells covering the whole extent of the optical galaxy. The complex radial velocity pattern indicates expansion velocities up to $60-75 \mathrm{~km} \mathrm{~s}^{-1}$. The majority of the supershells presents an interior OB-association, suggesting a physical link between them. Lozinskaya et al. (2003) identified three large ( $300 \mathrm{pc})$, bright $\mathrm{HI}$ supershells in the north-east part of the galaxy. They expand at $\sim 15 \mathrm{~km} \mathrm{~s}^{-1}$ and overlap with several smaller HII shells distributed along the walls of the HI supershells and are ionized by the young OB-associations. The location of the HII shells and OB-associations suggests that sequential star formation was triggered in this region by larger neutral hydrogen supershells. This implies that, despite a quiet history of star formation, the ISM of IC 1613 has complex gas kinematics and morphology which are linked to the embedded stellar population.

Here we present a study of the neutral hydrogen distribution and kinematics within this galaxy based on Very Large Array observations and compare them with our previous studies of the ionized gas and stellar content in this galaxy. We have found several giant $\mathrm{HI}$ rings and arc-like structures. The smallest ones present clear $21-\mathrm{cm}$ line splitting indicating that in these cases we observe expanding shells generated by the embedded OB-associations. The origin of the largest ones is not clear. In particular, the largest, $\sim 1 \mathrm{kpc}$ in diameter, structure contains several OB-associations of different ages which appear to be potential sources of energy. However we did not find any indication of a regular shell expansion that leads to an assumption that this structure may represent a case of the standing, pressure-confined shell. This initiated us to compare the observed parameters of the "main supershell" with the standard model based on the multiple winds and supernovae hypothesis. We found that the SNe hypothesis is in poor agreement with the observationally restricted rate of star formation that indicates a different origin of this large, kpc-scale structure.

The paper is organized as follows. In Sect. 2 we present the results of the observations, the procedures for the data reduction, discuss the neutral hydrogen distribution and the observed velocity field and compare this with properties of the ionized ISM and stellar content of the galaxy. In particular, we discuss 
properties of the largest HI structure $1 \mathrm{kpc}$ in diameter that is similar to the largest HI supershells detected in other galaxies. In Sect. 3 we discuss the standard superbubble model, present the results of numerical calculations and compare them with the observed parameters of the "main supershell", the largest HI structure detected in IC 1613. Section 4 summarizes our results.

\section{HI data: morphology, kinematics and their links with other galactic components}

Neutral hydrogen observations were made with the Very Large Array (VLA) of The National Radio Astronomy Observatory (NRAO) in configurations $\mathrm{B}, \mathrm{C}$ and $\mathrm{D}$. The application for the current observations was compiled by E. M. Wilcots. The first results, together with the brief discussion for data reduction, were presented in Lozinskaya et al. (2001).

The data cube has an angular resolution of 7'. $4 \times 7$ '. 0 , and a pixel size of $3^{\prime \prime} \times 3^{\prime \prime}$. This implies a linear resolution of $\sim 23 \mathrm{pc}$ at the adopted distance of $725 \mathrm{kpc}$. The spectral channel width is $2.57 \mathrm{~km} \mathrm{~s}^{-1}$. Our data cube allows us to derive the distribution and kinematics of the HI gas of the entire galaxy. To build up the position-velocity diagrams and the integrated $21-\mathrm{cm}$ line intensity map, the 40 velocity channels, from the 127 available, have been selected by the condition that the signal-to-noise ratio exceeds the $2 \sigma$ limit in three or more adjacent velocity channels (a windowing technique; see, for example, Thornley $\&$ Mundy 1997). The selected channels cover velocity interval from -279 to $-178 \mathrm{~km} \mathrm{~s}^{-1}$.

\subsection{The distribution of neutral hydrogen}

The integrated $21-\mathrm{cm}$ line flux density derived from our data is $482 \mathrm{Jy} \mathrm{km} \mathrm{s}^{-1}$. This implies that the total HI mass of the galaxy is $6.0 \times 10^{7} M_{\odot}$. This value is in good agreement with the single-dish measurements by Lake \& Skillman (1989; see Table II from their paper), despite a "negative bowl" in our data that leads to a negligible underestimation of the HI mass. An average 1D velocity dispersion of $5.9 \mathrm{~km} \mathrm{~s}^{-1}$ was measured from our data cube after accounting for the instrumental resolution. This corresponds to a $3 \mathrm{D}$ velocity dispersion $\sigma_{\text {ISM }}=10.2 \mathrm{~km} \mathrm{~s}^{-1}$. Lake \& Skillman (1989) adopted a somewhat larger value of $7.5 \mathrm{~km} \mathrm{~s}^{-1}$ for the $1 \mathrm{D}$ velocity dispersion. However, our data have better spectral and spatial resolutions ( $5 \mathrm{~km} \mathrm{~s}^{-1}$ versus $6.2 \mathrm{~km} \mathrm{~s}^{-1}$, and $7^{\prime \prime}$ versus $\left.45^{\prime \prime}\right)$.

The map of the $21-\mathrm{cm}$ line surface brightness is presented in Fig. 1a. The three brightest and most clearly defined shells (Shells I, II and III) in the N-E sector of the galaxy have diameters of 200-300 pc and are clearly related to the only region of noticeably enhanced star formation discovered so far in the galaxy (see Lozinskaya et al. 2003). Several more shells and arcs of similar sizes having lower surface brightness are clearly identified in Figs. 1a and 3a (e.g. Shells IV and V).

Besides these intermediate-size shells, one can easily recognize in Fig. 1 several larger arcs and ring-like structures which are similar in size and morphology to the supergiant shells revealed by Meaburn (1980) at the Large Magellanic Cloud. The most prominent structure seen in Fig. 1a coincides with the giant region of low surface brightness emission visible in the lower angular resolution map of Lake \& Skillman (1989). It is a hole surrounded by a thick HI ridge. The hole has a $5^{\prime}$ diameter $(\sim 1 \mathrm{kpc}$ diameter at the distance adopted for IC 1613) and is centered at $\mathrm{RA}(2000)=1^{\mathrm{h}} 4^{\mathrm{m}} 52^{\mathrm{s}}, \operatorname{Dec}(2000)=2^{\circ} 07^{\prime}$. The thickness of the HI ridge, $\Delta R$, is about $200 \mathrm{pc}$. The Northern, Eastern and Southern parts of the ring have a complicated morphology and a kinematical pattern suggesting a feedback from the local star formation. Shells II and III form the brightest part of the northern wall. The northwest sector of the ring appears to have a lower surface brightness and is not clearly identified in our map. Figure 1a also presents the locations of the OB-associations (shown by circles whose radii are proportional to the sizes of the OB-associations) taken from the list of Borissova et al. (2004). Figure 1b displays a population of superbubbles identified by Valdez-Gutiérrez et al. (2001). Hereafter the whole structure is referred to as the "main supershell".

We split the galaxy into a number of segments (Fig. 1c) and then calculated the mass of each segment by integration of the HI column density over the segment surface. The segment masses are presented in Table 1 . We associate the "main supershell" with segments 1, 2, 11, 14, 17 (Fig. 1c). The HI mass of the "main supershell" is then $2.8 \times 10^{7} M_{\odot}$, which corresponds to a total mass of $3.9 \times 10^{7} M_{\odot}$ when including helium.

Another example of a large shell-like structure is an elliptical hole in the HI distribution at the north of the main supershell surrounded by sectors 1, 5, 6, 7, 9 and 10 (Fig. 1c). One can also recognize several incomplete shells formed by sectors 10 , 11 and 12 , sectors $14,11,12,13$ and 15 , and sectors 13,15 and 16 at the northwest and west of the main supershell. The size of the shell to the north is similar to that of the "main supershell" but it has a smaller mass, $M_{\mathrm{HI}} \approx 9.7 \times 10^{6} M_{\odot}$.

\subsection{The ionized component, the stellar population, and the local SFR}

The examination of the distribution of the HII regions and superbubbles from the list of Valdez-Gutiérrez et al. (2001; Tables 4 and 5) shows that many of them are located inside the area encompassed by the contour of the "main supershell" or directly inside the HI ridge. Figure $1 \mathrm{~b}$ displays the boundaries of the superbubbles found by Valdez-Gutiérrez et al. (2001) superimposed on the HI image of the galaxy. Several superbubbles (R11, R12, R14, R15, R16 and R17) are located inside the "main supershell". R1, R4 and R6 seem to coincide with HI shells I, II and III identified by Lozinskaya et al. (2003). The rest are projected onto the HI ridge and seem to be associated with the extended region of star formation at the northeast of the galaxy.

The H $\alpha$ luminosities of the HII regions and superbubbles obtained by Valdez-Gutiérrez et al. (2001) allow us to estimate the mass of the ionized gas and the local star formation rate within the area encircled by the "main supershell". The mass of the ionized gas associated with superbubbles was calculated from the relation $M_{\mathrm{HII}}=3.3 \times 10^{-2} \sum\left(R_{i} / 1 \mathrm{pc}\right)^{3} n_{\mathrm{e}, i}\left(M_{\odot}\right)$, where radii of superbubbles, $R_{i}$, and electron number densities, 

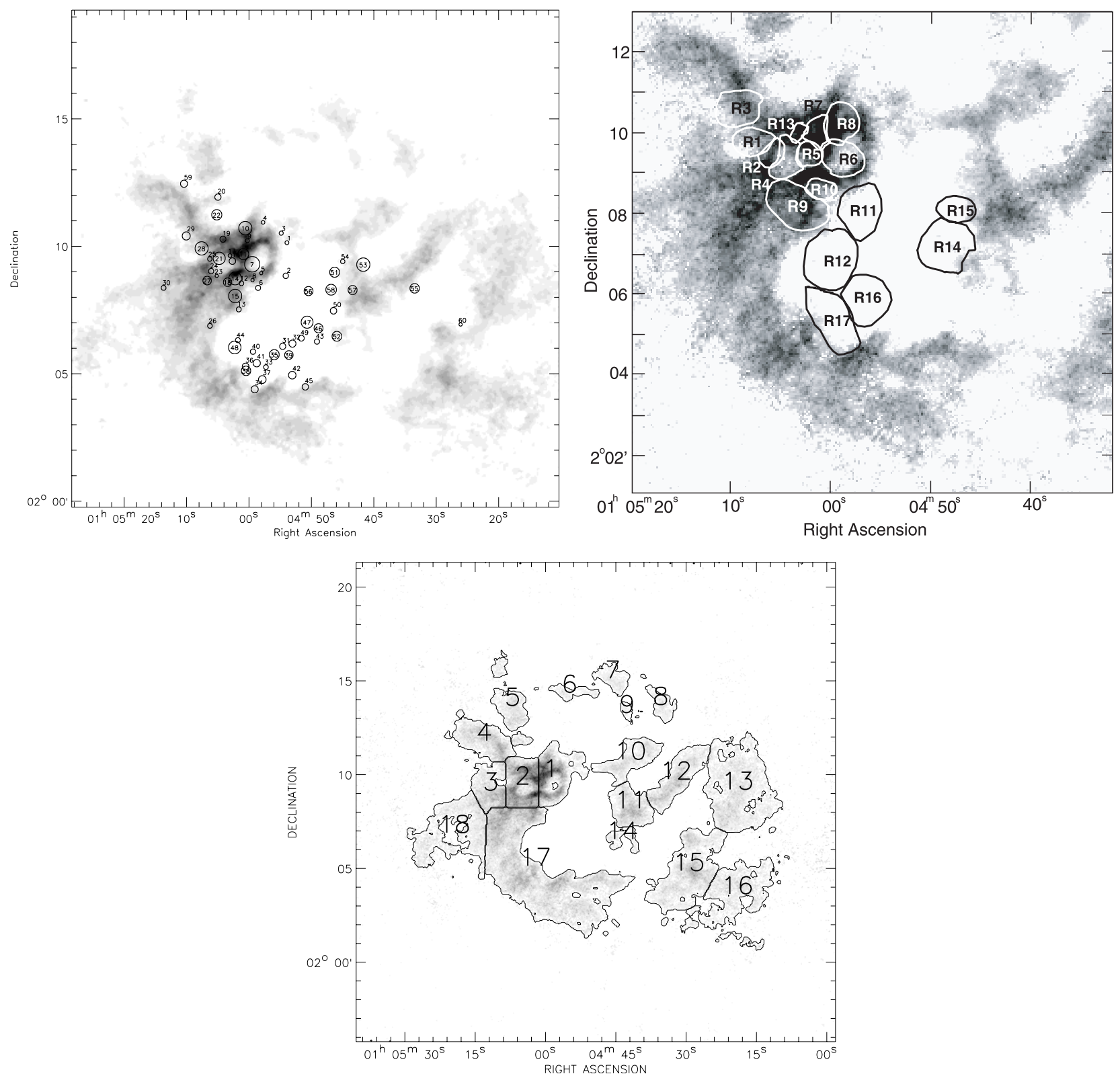

Fig. 1. The distribution of neutral hydrogen. Upper left: the $21-\mathrm{cm}$ line brightness map with OB associations from the list of Borissova et al. (2004) superposed. OB associations are shown by circles whose radii are proportional to their sizes. The coordinates and radii of associations are taken from the Table 1 of Borissova et al. (2004). Upper right: the 21-cm line brightness map of the southeast sector of the galaxy with the superbubbles of Valdez-Gutiérrez et al. (2001) superposed. Lower panel: the distribution of the HI column density throughout the galaxy split into a number of segments. In all panels north is at the top and east is on the left.

$n_{\mathrm{e}, i}$, were taken from Table 8 of Valdez-Gutiérrez et al. (2001), the thickness of the superbubble shells was assumed to be $1 / 12$ of their radii, and the contribution from the helium was taken into account. For the HII regions we assume a homogeneous density distribution. Then $M_{\mathrm{HII}}=3.3 \times 10^{-1} \sum\left(R_{i} / 1 \mathrm{pc}\right)^{3} n_{\mathrm{e}, i}$. The rms electron number densities were calculated from the relation $n_{\mathrm{e}, i}=\left(2.3 \times 10^{17} F_{\mathrm{H} \alpha, i} / \Theta_{i}^{2} R_{i}\right)^{1 / 2}$, where $\Theta_{i}$ is the equivalent angular diameter of the HII region expressed in square arc-seconds and $F_{\mathrm{H} \alpha, i}$ is the $\mathrm{H} \alpha$ flux in the units of $\mathrm{erg} \mathrm{cm}^{-2} \mathrm{~s}^{-1}$. The radii of the superbubbles and the HII regions were measured in parsecs. The total mass of the ionized component, $M_{\mathrm{HII}}=1.6 \times 10^{6} M_{\odot}$, is small in comparison with the HI mass of the main supershell and, therefore, we neglected its contribution in our calculations.

We then estimated the local SFR using the calibration given in Kennicutt (1998)

$\operatorname{SFR}\left(M_{\odot} \mathrm{yr}^{-1}\right)=7.9 \times 10^{-42} L_{\mathrm{H} \alpha}\left(\mathrm{erg} \mathrm{s}^{-1}\right)$,

adding up the luminosities of the superbubbles (R11, R12, R14, R15, R16 and R17 from the list of Valdez-Gutiérrez et al. (2001), see Fig. 1b) that are located inside the inner boundary of the "main supershell" and therefore may contribute to the dynamics of the supershell. We found that the reddening correction is small because, according to the 
Table 1. The distribution of the HI mass in the galaxy.

\begin{tabular}{lcccccc}
\hline \hline Segment & 1 & 2 & 3 & 4 & 5 & 6 \\
\hline Mass $\left(10^{6} M_{\odot}\right)$ & 5.37 & 6.17 & 2.39 & 2.86 & 1.70 & 0.38 \\
Segment & 7 & 8 & 9 & 10 & 11 & 12 \\
Mass $\left(10^{6} M_{\odot}\right)$ & 0.57 & 0.48 & 0.14 & 1.57 & 2.20 & 2.66 \\
Segment & 13 & 14 & 15 & 16 & 17 & 18 \\
Mass $\left(10^{6} M_{\odot}\right)$ & 4.95 & 0.34 & 4.46 & 3.60 & 14.02 & 3.18 \\
\hline Total mass $\left(10^{6} M_{\odot}\right): 57.0$ & & & & \\
\hline
\end{tabular}

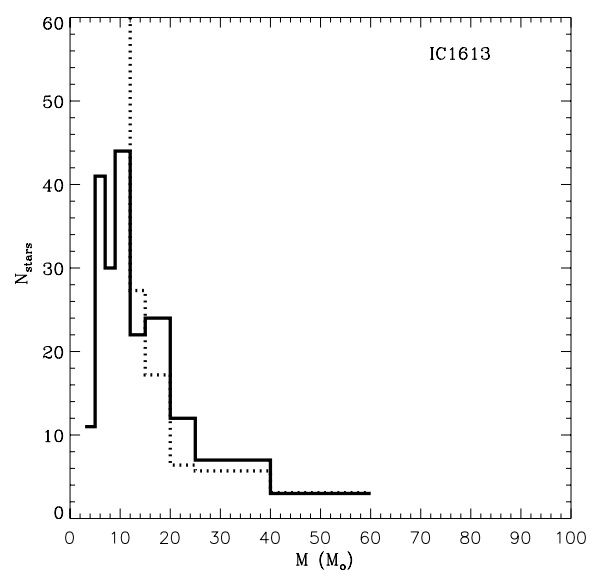

Fig. 2. The local star formation rate. The number of massive stars, members of OB-associations, which are located inside the main supershell (solid line) as compared to the number of massive stars derived from the constant star formation rate model (dotted line). The reasonable fit to the high mass top of the observed mass function requires the local rate of star formation to be $S F R_{\text {local }}=4.5 \times 10^{-4} M_{\odot} \mathrm{yr}^{-1}$.

NASA/IPAC-Extragalactic-Database (NED), $A_{\mathrm{v}}$ is only 0.083 mag. The $\mathrm{H} \alpha$ luminosity then is $3.24 \times 10^{37} \mathrm{erg} \mathrm{s}^{-1}$, and the local star formation rate, $S F R_{\text {local }}=2.6 \times 10^{-4} M_{\odot} \mathrm{yr}^{-1}$. This value has been compared with the SFR (Fig. 2) obtained from the analysis of stellar contents of OB-associations from the list of Borissova et al. (2004). The masses, the absolute visual magnitudes, $M_{\mathrm{v}}$, and the ages of OB-associations within the field of the main supershell are listed in Table 2.

The ages of the associations have been calculated from the observed color-magnitude diagrams which were converted into absolute magnitudes and the true colors for each of the associations. The foreground reddening was calculated for each star individually using the reddening free $Q$ parameter and the latest calibration equations of Massey et al. (2000) summarized in their Table 4. A distance modulus and a metallicity were adopted where $(m-M)_{0}=24.27 \pm 0.1$ (Dolphin et al. 2000), and $Z=0.004$ (Mateo 1998). The ages were then calculated by the best fit to the theoretical isochrones from the Geneva library (see Lejeune \& Schaerer 2001). The likely error of our method is 2-2.5 Myr.

In order to obtain the masses of individual stars and a generalized mass function we have used the procedure proposed by Massey et al. (2000), which allows the determination of the effective temperatures $\left(T_{\text {eff }}\right)$ and the bolometric corrections $(\mathrm{BC})$ for individual stars. The evolutionary tracks from Lejeune \& Schaerer (2001) for $Z=0.004$ were then used to establish mass
Table 2. OB-associations from Borissova et al. (2004) that are located within the field of the main supershell.

\begin{tabular}{|c|c|c|c|c|}
\hline OB-association & Number of O, B stars & $\begin{array}{l}\text { Mass } \\
\left(M_{\odot}\right)\end{array}$ & $\overline{M_{\mathrm{v}}}$ & $\begin{array}{r}\text { Age } \\
(\mathrm{Myr})\end{array}$ \\
\hline 31 & 6 & 62 & -4.48 & 25 \\
\hline 32 & 7 & 107 & -5.28 & 19 \\
\hline 33 & 10 & 109 & -4.18 & 24 \\
\hline 34 & 4 & 35 & -3.04 & 30 \\
\hline 35 & 17 & 236 & -5.90 & 16 \\
\hline 36 & 7 & 47 & -5.08 & 20 \\
\hline 37 & 7 & 59 & -5.47 & 18 \\
\hline 38 & 21 & 176 & -5.47 & 18 \\
\hline 39 & 6 & 48 & -4.91 & 21 \\
\hline 40 & 4 & 25 & -5.29 & 19 \\
\hline 41 & 5 & 35 & -4.03 & 25 \\
\hline 42 & 6 & 46 & -3.39 & 28 \\
\hline 43 & 4 & 17 & -3.42 & 28 \\
\hline 44 & 5 & 40 & -3.47 & 28 \\
\hline 45 & 5 & 34 & -4.72 & 22 \\
\hline 46 & 7 & 60 & -5.40 & 18 \\
\hline 47 & 7 & 41 & -4.82 & 21 \\
\hline 49 & 4 & 19 & -3.65 & 27 \\
\hline 50 & 4 & 22 & -2.86 & 34 \\
\hline 51 & 5 & 35 & -3.61 & 28 \\
\hline 52 & 6 & 36 & -3.88 & 28 \\
\hline 53 & 7 & 52 & -3.70 & 26 \\
\hline 54 & 6 & 28 & -4.54 & 23 \\
\hline 55 & 6 & 25 & -4.80 & 21 \\
\hline 56 & 4 & 28 & -5.41 & 18 \\
\hline 57 & 4 & 14 & -3.72 & 27 \\
\hline 58 & 7 & 38 & -5.00 & 20 \\
\hline
\end{tabular}

intervals. The local SFR derived from the comparison of this mass function with the constant star formation rate model is $S F R_{\text {local }}=4.5 \times 10^{-4} M_{\odot} \mathrm{yr}^{-1}$.

Obviously, these values only give a lower limit to the star formation rate within the galactic area covered at the current epoch by the "main supershell". The mass function obtained from the Borissova et al. (2004) data is incomplete at the low mass end and does not account for the field stars. The local SFR derived from the $\mathrm{H} \alpha$ data is also related to the current epoch and does not consider the diffuse $\mathrm{H} \alpha$ emission. Nevertheless our estimates are reasonable when compared to the mean star formation rate over the galaxy. Therefore we adopt them as a likely limit to the SFR within the field of the main supershell.

\subsection{The HI kinematics}

In order to study the kinematics of the interstellar medium (ISM) in IC 1613 we obtained position-velocity diagrams in more than 30 directions. The orientations of several scans and the corresponding position-velocity diagrams are presented in Fig. 3.

The kinematics of Shells I, II and III were discussed by Lozinskaya et al. (2003). Shells II and III have expansion velocities of $12-18 \mathrm{~km} \mathrm{~s}^{-1}$ and kinematical ages 5.3-5.6 Myr. The expansion velocity of Shell I could not be measured, while its size is similar to that of Shells II and III. Shells IV and V 

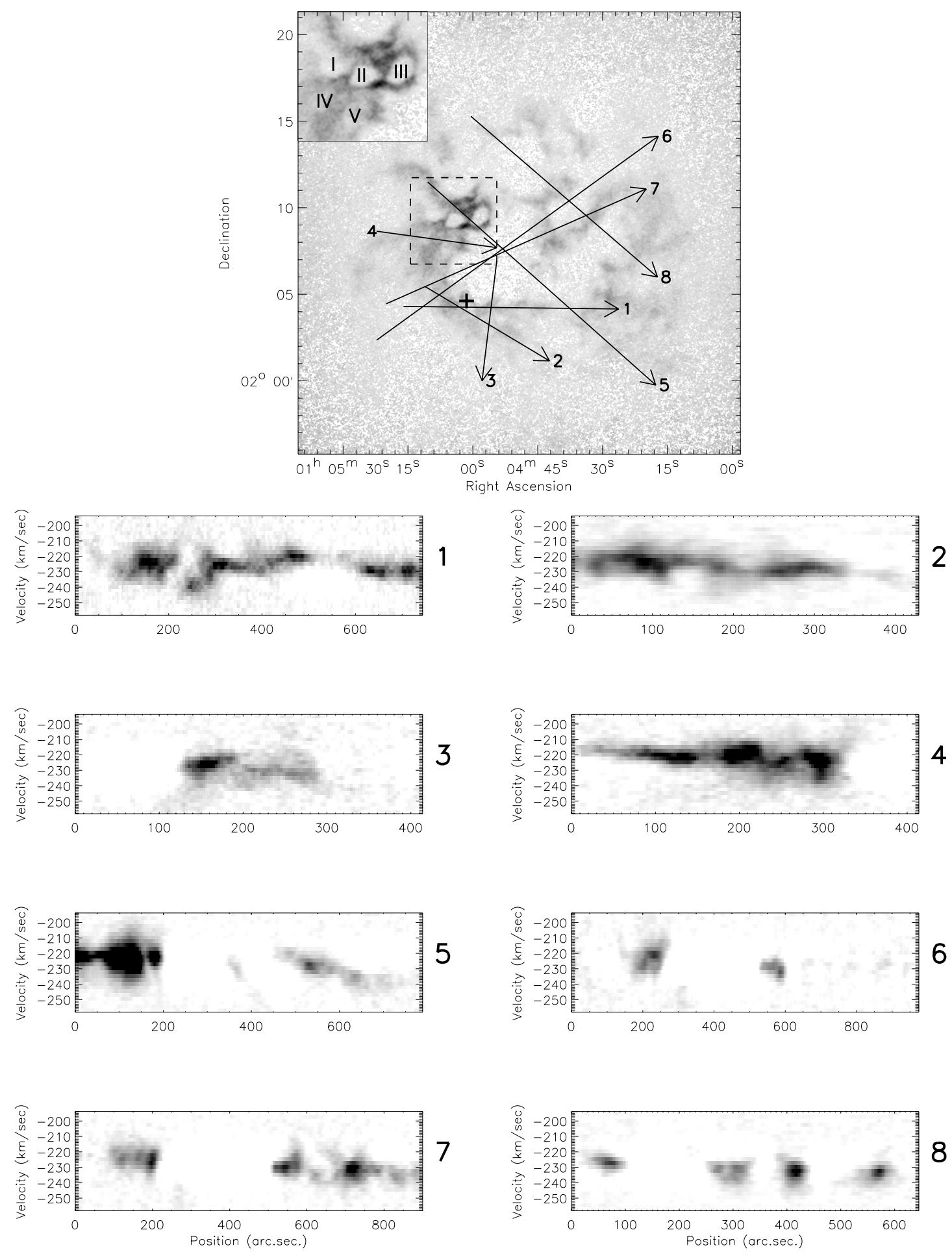

Fig. 3. The neutral gas kinematics. Upper panel displays orientations of cuts used to study the neutral gas kinematics. Cuts are identified by numbers. The region of active star formation in the northeast sector of the galaxy is shown in the upper left-hand box. The position of the WO star is marked by a cross. Panels at the bottom represent the position-velocity diagrams along each cut indicated at the upper panel. All velocities are heliocentric.

mentioned in the previous section are much fainter and their properties are harder to determine than those of Shells I, II and III. Their radii are about $150-200 \mathrm{pc}$. The position-velocity diagram taken across the eastern part of the "main supershell" (cut 4 at Fig. 3a) reveals an offset of approximately of $15 \mathrm{~km} \mathrm{~s}^{-1}$ between the regular rotation velocity of the galactic gas (which is in between $-225 \mathrm{~km} \mathrm{~s}^{-1}$ and $-235 \mathrm{~km} \mathrm{~s}^{-1}$ in this region) and the maximum values found in the field of the shells. This is $-215 \mathrm{~km} \mathrm{~s}^{-1}$ for shell IV (at 170"-230" along the cut), and $-220 \mathrm{~km} \mathrm{~s}^{-1}$ at the field of Shell V (at $250^{\prime \prime}-315^{\prime \prime}$ along the 
cut). These offsets together with the characteristic shapes of local velocity ellipses suggest that the expansion velocities of both of the shells are around $15 \mathrm{~km} \mathrm{~s}^{-1}$.

The position-velocity diagram taken along a shell formed by sectors 10,11 and 12 also indicates a regular shell expansion (see cuts 7 and 8 at Fig. 3, at 550"-700" and 300" $-400^{\prime \prime}$ along the cuts). The expansion velocity is around $13 \mathrm{~km} \mathrm{~s}^{-1}$. The size of this shell is $3^{\prime}$ that corresponds to a linear diameter, $D=640 \mathrm{pc}$, and the HI mass is $6.4 \times 10^{6} M_{\odot}$ (see Table 1 ). Other giant structures at the north and northwest of the "main supershell" show no evidences of regular shell expansion.

The "main supershell" was cut in 12 directions. We did not find any indication of regular expansion of the "main supershell" in any of the cuts. Only local gas perturbations were identified in some sectors of the "main supershell" (e.g. at the positions corresponding to $115^{\prime \prime}-200^{\prime \prime}$ of cut $2,135^{\prime \prime}-180^{\prime \prime}$ of cut $3,430^{\prime \prime}-630^{\prime \prime}$ of cut 5 and $90^{\prime \prime}-210^{\prime \prime}$ of cut 7). The observed local velocities are in the range $215-245 \mathrm{~km} \mathrm{~s}^{-1}$. The best example of the local high velocity flow is observed in the vicinity of the WO star which is located at the southeast wall of the "main supershell" (see Lozinskaya et al. 2001). In this region we probably observe the blow-out of the WO stellar wind from the shell into the low density bubble interior. The local velocities observed in this region (see Fig. 3b, at the positions corresponding to $190^{\prime \prime}-300^{\prime \prime}$ along the cut 1 ) fall into the range $-205 \mathrm{~km} \mathrm{~s}^{-1}--250 \mathrm{~km} \mathrm{~s}^{-1}$. We believe that other arc-shaped structures seen in the inner part of Segment 17 result from the collective feedback given by the associations 26, 34, 36, 37, $38,42,45$ of Borissova et al. (2004), which are located near the ridge of the "main supershell". The expansion velocities of the local arcs are less than or equal to $25 \mathrm{~km} \mathrm{~s}^{-1}$.

The absence of regular expansion and the large thickness of the "main supershell" led us to conclude that if this structure results from the combined action of the embedded groups of massive stars, then the main supershell has already reached its standing position and is now confined by the external gas pressure (see Koo \& McKee 1992).

\section{The multiple SNe scenario}

In the standard SNe scenario, the shell expansion is supported by a high thermal pressure provided by multiple supernova explosions and stellar winds from massive stars recently formed inside the superbubble volume. In the case of an homogeneous ISM and a constant input rate of mechanical energy, the time evolution of the radius, $R$, the expansion velocity, $V$, and the mass, $M$, of the shell are given by (see Bisnovatyi-Kogan \& Silich 1995, and references therein):

$$
\begin{aligned}
R & =\left(\frac{125}{154 \pi}\right)^{1 / 5}\left(\frac{L_{\text {mech }}}{\rho_{\mathrm{ISM}}}\right)^{1 / 5} t^{3 / 5} \\
V & =\frac{3}{5} \frac{R}{t} \\
M & =\frac{4 \pi}{3} R^{3} \rho_{\mathrm{ISM}} ;
\end{aligned}
$$

where $t$ is the bubble age, $L_{\text {mech }}$ is the rate of the mechanical energy deposited by $\mathrm{SNe}$ explosions and stellar winds and $\rho_{\mathrm{ISM}}$ is the density of the ISM. Eliminating the evolutionary time $t$ and the ISM density $\rho_{\text {ISM }}$ from Eqs. (2)-(4), one can find the mechanical power, $L_{\text {mech }}$, required to create a shell with radius $R$, mass $M$ and expansion velocity $V$ :

$$
L_{\text {mech }}=\frac{77}{18} \frac{M V^{3}}{R}=2.8 \times 10^{38} \frac{M_{7} V_{10}^{3}}{R_{100}} \mathrm{erg} \mathrm{s}^{-1},
$$

where $M_{7}$ is the mass of the shell in units of $10^{7} M_{\odot}, V_{10}$ is the expansion velocity in units of $10 \mathrm{~km} \mathrm{~s}^{-1}$ and $R_{100}$ is the radius of the shell in units of $100 \mathrm{pc}$.

The transition to the pressure-confined stage occurs when the velocity of the shock approaches the ambient gas turbulent speed, $V=\sigma_{\text {ISM }}$. At that time the shell begins to expand in both directions from the stand-off radius, $R_{\text {stand }}$, with the local sound speed, $c_{\mathrm{sh}}$. This may be consistent with the irregular boundary of the main supershell and with the standard scenario. If this is the case, it takes the shell $\sim 0.5 \Delta R / c_{\mathrm{sh}} \geq 10^{7} \mathrm{yr}$ to reach the observed thickness. Thus the detected configuration may be observed for a good fraction $(\sim 1 / 4)$ of the total evolutionary time, $t$. The stand-off radius is $R_{\text {inner }}<R_{\text {stand }}<R_{\text {out }}$, where $R_{\text {inner }}$ is the inner and $R_{\text {out }}$ is the outer radius of the shell. We assume that

$R_{\text {stand }}=R_{\text {inner }}+\frac{\Delta R}{2}=600 \mathrm{pc}$

where $\Delta R \approx 200 \mathrm{pc}$ is the mean thickness of the shell. For the rate of mechanical energy we then obtain: $L_{\text {mech }} \approx 1.9 \times$ $10^{38} \mathrm{erg} \mathrm{s}^{-1}$. This energy input rate requires a coeval star cluster of approximately $10^{4} M_{\odot}$ with a Salpeter initial mass function with a lower and upper mass limits of $1 M_{\odot}$ and $100 M_{\odot}$. We do not see stellar remnants of such massive clusters in the field of view of the "main supershell". On the contrary, OB-associations found inside the ring have a wide range of ages (see Table 2) that indicates that in the region encompassed by the main supershell the star formation lasts for more than 30 Myr. This suggests that a continuous star formation rate would be a better approximation for the star formation activity that occurred in this region.

In this case the rate of mechanical energy cannot be approximated by a constant value during the whole evolution. In such a scenario the maximum value is reached 30 Myr after the beginning of star formation (see Leitherer \& Heckman 1995). On the other hand the thermal pressure of the interstellar gas also reduces the shell radius below the standard value (Oey \& García-Segura 2004). The calculated energy may be also affected by the deviation of the ISM density from a homogeneous distribution.

To take into consideration these effects and to obtain a realistic estimate for a SFR that is required to build up a shell of similar to the observed structure, we have provided several numerical runs using for calculations our 2.5D Lagrangian scheme based on the thin layer approximation (see Bisnovatyi-Kogan \& Silich 1995; Silich \& Tenorio-Tagle 1998). As an input model we adopt an exponential galactic disk with a constant velocity dispersion $\sigma=10.2 \mathrm{~km} \mathrm{~s}^{-1}$,

$\rho_{\text {ISM }}=\rho_{0} \exp \left(-|z| / H_{z}\right)$, 
where $\rho_{0}$ is the midplane ISM density. It was assumed that the disk is embedded in a low density gaseous halo whose density was taken to be $n_{\text {halo }}=10^{-5} \mathrm{~cm}^{-3}$. We neglected the small shell distortion (Palouš et al. 1990; Silich 1992) provided by the slow galactic rotation. The Z-component of the gravitational field was calculated from the equilibrium conditions. The mass and energy deposition rates were calculated from our simplified starburst model (see Silich et al. 2002) assuming that the SFR remains constant until the shell waist attains the observed radius. The SFR and the midplane ISM density $\rho_{0}$ were then calculated by iterations from the condition that the expansion velocity of the shell waist becomes equal to the ISM turbulent velocity and the calculated mass of the shell approaches the observed value when the radius of the shell waist reaches $600 \mathrm{pc}$.

The calculations have been provided for two different (thin and thick) galactic disk models with the characteristic scaleheights of the exponential disk $H_{z}=200 \mathrm{pc}$ and $H_{z}=500 \mathrm{pc}$, respectively. Figure 4 presents the evolutionary sequence of superbubble shapes for these two cases.

In both cases the bubbles grow faster in the direction of the steepest density gradient. In the case of the thin disk, the superbubble already reaches a typical hour-glass form after $20 \mathrm{Myr}$ (Fig. 4a). Soon the shell accelerates rapidly into the low-density galactic halo and the top of the shell is disrupted under the action of Rayleigh-Taylor instabilities. At this age ( $\approx 20 \mathrm{Myr})$, the top of the shell reaches approximately three characteristic $Z$-heights of the interstellar gas distribution. The remnant is then able to "blow out", driving its high temperature gas between the fragments into the halo of the host galaxy (Silich \& Tenorio-Tagle 1998), and to establish the cone-like structure seen in Fig. 4b. The bubble then loses its thermal pressure and the waist of the shell reaches its standing position at the age $t \approx 34$ Myr. Despite the large size of the cone, the bulk of the swept-up material is located within a thin cylindrical segment around the base of the cone. In this particular case, about $50 \%$ of the mass of the remnant is concentrated inside a $400 \mathrm{pc}$ (200 pc above and $200 \mathrm{pc}$ below the midplane of the galaxy) segment.

To fit the observed parameters of the main supershell the midplane gas number density should be $n_{0} \approx 2.8 \mathrm{~cm}^{-3}$ and the star formation rate $S F R \approx 7.5 \times 10^{-3} M_{\odot} \mathrm{yr}^{-1}$. This value is more then an order of magnitude larger than the local star formation rate, $S F R_{\text {local }} \approx(3-4) \times 10^{-4} M_{\odot} \mathrm{yr}^{-1}$, derived from the analysis of our $\mathrm{H} \alpha$ data and stellar population of the embedded OB-associations, and approximately three times larger than the average star formation rate obtained by Cole et al. (1999) for the entire galaxy. Thus the thin-disk model is completely inconsistent with the the standard SNe scenario.

In the thick disk case the shell remains almost round after 20 Myr of expansion. Even at the end of the calculations, at $t \approx 37 \mathrm{Myr}$, the shell is only slightly distorted by the ISM density gradient (see Fig. 4d). The Rayleigh-Taylor instabilities never disrupt the top of the shell and the hot gas remains bound within the cylindrically-shaped remnant slowly expanding in the $Z$-direction. To fit the observed parameters (the stand-off radius and the mass of the shell), the mid-plane density of the disk should be $\approx 1.4 \mathrm{~cm}^{-3}$ and the star formation rate must be about $2.3 \times 10^{-3} M_{\odot} \mathrm{yr}^{-1}$. This value significantly ( $\sim 5$ times)
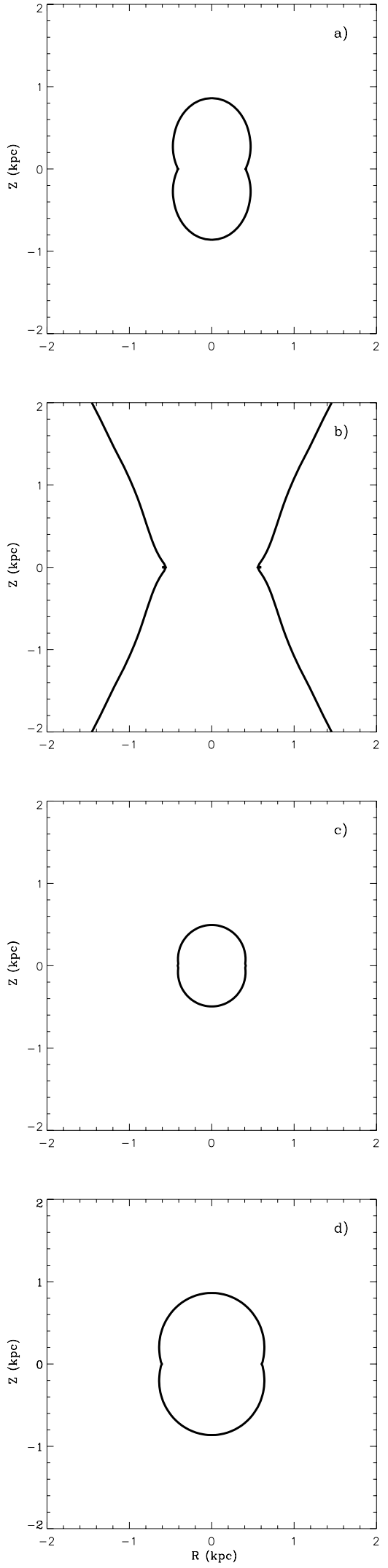

Fig. 4. The shape of the supershell. Panels a), b) and c), d) present the results of the calculations for the thin $\left(H_{z}=200 \mathrm{pc}\right)$ and thick $\left(H_{z}=\right.$ $500 \mathrm{pc}$ ) disk models, respectively. The ages of the shells in panels a) and c) are similar ( $\approx 20 \mathrm{Myr})$. Panels b) and d) present supershells at the moment when they reach their standing positions at the midplane of the galaxy. 
exceeds our limit to the local star formation rate and is only slightly below the mean star formation rate obtained for the entire galaxy. There are also no indications on a massive single stellar cluster that may support shell expansion down to the observed radius. This leads us to the conclusion that multiple SNe hypothesis is inconsistent with the observed parameters of the "main supershell" also in the case of the thick disk ISM distribution. Some different mechanism (e.g. the outward force initiated by the radiation pressure of the field stars, Elmegreen $\&$ Chiang 1982) is required to build up the observed structure.

\section{Conclusions}

Here we presented the results of our high resolution study of neutral hydrogen distribution and kinematics in a Local Group member, the dwarf irregular galaxy IC 1613 and compared them to our previous examination of the stellar population and ionized component of the ISM in this galaxy. The analysis of the $21 \mathrm{~cm}$ line data cube reveals a complicated, highly inhomogeneous structure of the interstellar medium with a number of HI holes, shells and arc-like structures of different sizes and expansion velocities.

We found that smaller shells with diameters of a few hundred parsecs tend to expand with velocities $10 \mathrm{~km} \mathrm{~s}^{-1} \leq V \leq$ $20 \mathrm{~km} \mathrm{~s}^{-1}$ whereas the largest structures do not present evidences of global expansion. The only exception is the large incomplete shell at the northwest part of the galaxy with mass $\sim 6.4 \times 10^{6} M_{\odot}$ and expansion velocity $V \approx 13 \mathrm{~km} \mathrm{~s}^{-1}$. The best example of the large standing structure is an HI hole $1 \mathrm{kpc}$ in diameter surrounded by a $2.8 \times 10^{7} M_{\odot}$ neutral hydrogen ring centered at $\operatorname{RA}(2000)=1^{\mathrm{h}} 4^{\mathrm{m}} 52^{\mathrm{s}}, \operatorname{Dec}(2000)=2^{\circ} 07^{\prime}$.

There are many OB-associations of different ages inside the hole whose energetics potentially may be responsible for the development of this structure. To examine this hypothesis we have compared the energy required by the multiple supernovae model with that inferred from the star formation rate derived from the analysis of the embedded stellar population and $\mathrm{H}_{\alpha}$ emission. We found that parameters of the observed structure and the level of the star formation activity detected inside the region encompassed by the contour of the "main supershell" are inconsistent with the SNe hypothesis. This indicates that, as it has been revealed in the past for the largest HI holes in HoII and LMC (Rhode et al. 1999; Broun et al. 1997), some different mechanism is required to build up the largest kpc-size HI structure that is detected in IC 1613.

Acknowledgements. The authors thank William Wall and Guillermo Tenorio-Tagle for careful reading of the manuscript and suggestions and our referee, Dr. A. Burkert, for his critical comments. We also greatly appreciate help from the stuff of The National Radio Astronomy Observatory (NRAO) where the observations were performed. NRAO is part of the National Science Foundation (USA) and is operated by the Association of Universities Inc. under a contract with the NSF. This study was supported by CONACYT México research grants 47534-F and 40095-F, by DGAPA-UNAM grant IN120802, by Russian Foundation for Basic Research (projects 02-02-06048 and 04-02-16042). A.V.M. also wishes to thank the Russian Science Support Foundation.

\section{References}

Bisnovatyi-Kogan, G. S., \& Silich, S. A. 1995, Rev. Mod. Phys., 67, 661

Borissova, J., Kurtev, R., Georgiev, L., \& Rosado, M. 2004, A\&A, 413, 889

Brinks, E., \& Bajaj, E. 1986, A\&A, 169, 14

Broun, J. M., Bomans, D. J., Will, J., \& de Boer, K. S. 1997, A\&A, 328,167

Bureau, M., \& Carignan, C. 2002, AJ, 123, 1316

Cole, A. A., Tolstoy, E., Gallagher, J. S., et al. 1999, AJ, 118, 1657

Davies, R. D., \& Tovmassian, H. M. 1963, MNRAS, 127, 45

Dib, S., \& Burkert, A. 2004, Ap\&SS, 292, 135

Dolphin, A., Saha, A., Skillman, E. D., Tolstoy, E., et al. 2001, ApJ, 550,554

Dyson, J. E., \& de Vries, J. 1972, A\&A, 20, 223

Efremov, Y. N., Ehlerová, S., \& Palouš, J. 1999, ApJ, 350, 457

Ehlerová, S., Palouš, J., \& Wünsch, R. 2004, Ap\&SS, 289, 279

Elmegreen, B. G., \& Chiang, W.-H. 1982, ApJ, 253, 666

Freedman, W. 1988a, ApJ, 326, 691

Freedman, W. 1988b, AJ, 326, 96

Georgiev, L., Borissova, J., Rosado, M., et al. 1999, A\&AS, 134, 21

Heiles, C. 1984, ApJS, 55, 585

Hunter, D. A., Hawley, W. N., \& Gallagher, J. S. 1993, AJ, 106, 1797

Kennicutt, R. C. 1998, ARA\&A, 36, 189

Kim, S., Dopita, M. A., Stavelet-Smith, L., \& Bessel, M. 1999, A\&A, 350,230

Koo, B.-C., \& McKee, C. F. 1992, ApJ, 388, 93

Lake, G., \& Skillman, E. D. 1989, AJ, 98, 1274

Leitherer, C., \& Heckman, T. M. 1995 , ApJS, 96, 9

Lejeune, T., \& Schaerer, D. 2001, A\&A, 365, 535

Lozinskaya, T., Moiseev, A., Afanasiev, V., Wilcots, E., \& Goss, W. M. 2001, Astron. Rep., 45, 417

Lozinskaya, T., Moiseev, A., \& Podorvanyuk, N. 2003, Astron. Lett., 29,77

Massey, P., Waterhouse, E., \& DeGioia-Eastwood, K. 2000, AJ, 119, 2214

Mateo, M. 1998, ARA\&A, 36, 435

Meaburn, J. 1980, MNRAS, 192, 365

Oey, M. S., \& García-Segura, G. 2004, ApJ, 613, 302

Palous, J., Franco, J., \& Tenorio-Tagle, G. 1990, A\&A, 227, 175

Perna, R., \& Gaensler, B. M. 2004, ApJ, 606, 326

Puche, D., Westpfahl, D., Brinks, E., \& Roy, J.-R. 1992, AJ, 103, 1841

Rhode, K. L., Salzer, J. J., Westpfahl, D., \& Radice, L. A. 1999, AJ, 118,323

Silich, S. A. 1992, Ap\&SS, 195, 317

Silich, S., \& Tenorio-Tagle, G. 1998, MNRAS, 299, 249

Silich, S., Tenorio-Tagle, G., Muñoz-Tuñón, C., \& Cairos, L. M. 2002, AJ, 123, 2438

Skillman, E. D., Tolstoy, E., Cole, A. A., et al. 2003, ApJ, 596, 253

Stewart, S. G., Fanelli, M. N., Byrd, G. G., et al. 2000, ApJ, 529, 201

Stil, J. M., \& Israel, F. P. 2002, A\&A, 389, 29

Tenorio-Tagle, G. 1981, A\&A, 94, 338

Tenorio-Tagle, G., \& Bodenheimer, P. 1988, ARA\&A, 26, 145

Thornley, M. D., \& Mundy, L. G. 1997, ApJ, 490, 682

Valdez-Gutierrez, M., Rosado, M., Georgiev, L., Borissova, J., \& Kurtev, R. 2001, A\&A, 366, 35

van der Hulst, T. 1996, in ASP Conf. Ser., 106, ed. E. D. Skillman, 47

Vorobyov, E. I., \& Basu, S. 2005, A\&A, 431, 451

Vorobyov, E. I., Klein, U., Shchekinov, Yu. A., \& Ott, J. 2004, A\&A, 413, 939

Weaver, R., McCray, R., Castor, J., Shapiro, P., \& Moore, R. 1977, ApJ, 218, 377 